Brief article

\title{
Even at 4 months, a labial is a good enough coronal, but not vice versa
}

\author{
Sho Tsuji ${ }^{\text {a,b,*,1 }}$, Reiko Mazuka ${ }^{\mathrm{c}, \mathrm{d}, 2}$, Alejandrina Cristia ${ }^{\mathrm{e}, 3}$, Paula Fikkert ${ }^{\mathrm{a}, 4}$ \\ a Radboud University Nijmegen, The Netherlands \\ ${ }^{\mathrm{b}}$ International Max-Planck Research School for Language Sciences, The Netherlands \\ ${ }^{\mathrm{c}}$ RIKEN Brain Sciences Institute, Japan \\ ${ }^{\mathrm{d}}$ Duke University, United States \\ e Laboratoire de Sciences Cognitives et Psycholinguistique, (ENS, EHESS, CNRS), Département d'Etudes Cognitives, Ecole Normale Supérieure, PSL \\ Research University, France
}

\section{A R T I C L E I N F O}

\section{Article history:}

Received 4 October 2013

Revised 14 October 2014

Accepted 22 October 2014

\section{Keywords:}

Infant

Language acquisition

Speech perception

Perceptual asymmetry

Cross-linguistic comparison

\begin{abstract}
A B S T R A C T
Numerous studies have revealed an asymmetry tied to the perception of coronal place of articulation: participants accept a labial mispronunciation of a coronal target, but not vice versa. Whether or not this asymmetry is based on language-general properties or arises from language-specific experience has been a matter of debate. The current study suggests a bias of the first type by documenting an early, cross-linguistic asymmetry related to coronal place of articulation. Japanese and Dutch 4- and 6-month-old infants showed evidence of discrimination if they were habituated to a labial and then tested on a coronal sequence, but not vice versa. This finding has important implications for both phonological theories and infant speech perception research.
\end{abstract}

(c) 2014 Elsevier B.V. All rights reserved.

\section{Introduction}

The special status attributed to coronal place of articulation in the phonologies of the world (Paradis \& Prunet, 1991) has intrigued phonologists for decades. Indeed, coronals (sounds articulated with the tongue tip or blade) show distinct characteristics, such as a high frequency of

\footnotetext{
* Corresponding author at: Max-Planck Institute for Psycholinguistics, Wundtlaan 1, 6525 XD Nijmegen, The Netherlands. Tel.: +31 631939717.

E-mail addresses: tsujish@gmail.com, sho.tsuji@mpi.nl (S. Tsuji), mazuka@brain.riken.jp (R. Mazuka), alecristia@gmail.com (A. Cristia), P. Fikkert@let.ru.nl (P. Fikkert).

${ }^{1}$ Present address: Laboratory for Language Development, RIKEN Brain Sciences Institute, 2-1 Hirosawa, Wako, Saitama 351-0198, Japan.

${ }^{2}$ Address: Laboratory for Language Development, RIKEN Brain Sciences Institute, 2-1 Hirosawa, Wako, Saitama 351-0198, Japan.

${ }^{3}$ Address: Laboratoire de Sciences Cognitives et Psycholinguistique, Pavillon Jardin, 29, rue d'Ulm, 75005 Paris, France.

4 Address: Center for Language Studies, Radboud University Nijmegen, Erasmusplein 1, 6525 HT Nijmegen, The Netherlands.
}

occurrence between and within languages (Maddiesson, 1984), and a proneness to undergo phonological processes such as place assimilation (Chomsky \& Halle, 1968). This special status would also affect speech processing in the form of a perceptual asymmetry, but whether or not this asymmetry is based on language-general properties (thus, is independent from a listener's language-specific experience) or arises from language-specific experience (thus, changes as a function of experience with a specific language) has been a matter of debate. The current study suggests that language-general properties underlie the special status of coronals based on evidence from young infants.

To highlight the issues being debated, we will introduce two accounts that capture perceptual asymmetries in adults. The Featurally Underspecified Lexicon (FUL; Lahiri \& Reetz, 2010) posits perceptual asymmetries independent of experience with a specific language. It assumes sparse and abstract lexical representations in which not all phonological features are specified. Coronal place of articulation is considered the default place, and 
consequently, it is underspecified in the mental lexicon. This predicts perceptual asymmetries such that labial mispronunciations of coronals (e.g., [bol] for $/ \mathrm{d} b \mathrm{l} /$ ) do not produce a mismatch ([bol] is accepted as an instance of $/ \mathrm{dol} /$ ), but coronal mispronunciations of labials do ([dol] is not accepted for $/ \mathrm{bol} /$ ). The results of numerous perceptual experiments are consistent with this prediction: labial mispronunciations prime coronal target words, but not vice versa, in cross-modal priming (Lahiri \& Reetz, 2002). Similarly, event-related potential (ERP) studies have shown smaller ERPs to labial mispronunciations of coronals than vice versa (e.g., Cornell, Lahiri, \& Eulitz, 2013).

Other work fails to support the predictions of FUL. Bonte, Mitterer, Zellagui, Poelmans, and Blomert (2005) reported smaller ERPs in response to a coronal-to-labial change compared to the opposite direction, but only when the non-words containing labials had a higher phonotactic probability than those containing coronals. With opposite phonotactic probabilities, this asymmetry reversed. In a series of three eye-tracking experiments, Mitterer (2011) found no evidence for asymmetric perception consistent with FUL, while a fourth experiment found an asymmetry predicted by phonotactic probability, but not FUL (but see Cornell et al., 2013). Based on this, Mitterer (2011) suggested an Optimal Perception account: Asymmetries reflect listeners' familiarity with the phonotactic probability of the input, such that listeners are biased towards accepting a frequent pattern more often than an infrequent one. Given that coronals are very frequent, predictions from this account align with those made by FUL in many cases, but based on the fundamentally different premise of language-specific experience.

To what extent the perceptual asymmetries are independent of language experience can, however, not be conclusively answered based on studies testing adult listeners with rich language experience, even more so because the majority of evidence comes from native speakers of Germanic languages. One way to address this debate is to assess whether the asymmetry is present already in prelexical infants. Based on earlier research, we can assume that infants' perception is not tuned to native consonant categories and phonotactic probabilities until after 6 months of age (cf. Kuhl, 2004, for an overview). Thus, early asymmetries would be independent of the extensive phonotactic experience judged necessary by the Optimal Perception account. A first piece of evidence for such an early asymmetry already exists: 6-month-old Dutch infants were able to detect the change from /pa:n/ to / ta:n/, but not vice versa (Dijkstra \& Fikkert, 2011). Nonetheless, as more sensitive methods appear, age of acquisition is constantly being pushed down (e.g., Bergelson \& Swingley, 2012). We built an even stronger test of the language-independent nature of such perceptual asymmetries by measuring discrimination at two early ages (4 and 6 months), in two language backgrounds with markedly different phonologies, namely Dutch and Japanese. Japanese is illuminating because, unlike Dutch, coronal is not the most frequent place of articulation for plosives (across both token and type frequency counts in content words; Tsuji, Nishikawa, \& Mazuka, 2010). Therefore, coronal as the default place and as the most frequent place are not confounded. If experience-independent perceptual biases can indeed contribute to perceptual asymmetries, a coronal-labial asymmetry should be observed in infants regardless of age and language background.

\section{Methods}

\subsection{Participants}

Sixteen 4-month-old Dutch (range 3.7-4.5 months, 8 females) and sixteen 4-month-old Japanese (4.05.0 months, 6 females) infants, as well as sixteen 6month-old Dutch (range 6.4-6.9 months, 10 females) and sixteen 6-month-old Japanese (range 6.1-7.0 months, 9 females) infants were included in the final sample. Dutch infants were recruited and tested in the Netherlands, and Japanese infants in Japan. All infants were healthy fullterm infants, raised in monolingual native Dutch or Japanese speaking households. Caregivers gave written consent to participate.

Twenty-nine additional infants were tested but not included in the final sample because of failure to reach the habituation criterion ( 7 Dutch, 1 Japanese), obscured view on the infant's eyes (1 Dutch), failure to look at the screen after experiment commencement (2 Japanese), fussiness or crying (8 Dutch, 10 Japanese).

\subsection{Stimuli}

Stimuli involved a labial-coronal contrast in their wordmedial consonant cluster (/ompa-onta/). In addition to the small burst and fast transitions found in word-initial plosives, e.g. /pa:n-ta:n/, our stimuli contain rich formant transitions into the nasals' place and some information in the nasal murmur. That 4.5-month-old infants are able to distinguish labial from coronal nasals in /ompa-onpa/ has been demonstrated previously (Jusczyk, Smolensky, \& Alloco, 2002). Both sequences are phonotactically legal in both Dutch and Japanese, although the frequency of / onta/ is higher than that of $/ \mathrm{ompa}$ / in Dutch (ratio of words containing /onta/ to /ompa/: 7.23; based on CELEX: Baayen, Piepenbrock, \& Gulikers, 1995), whereas the opposite is true in Japanese (ratio of words containing /onta/ to / ompa/: 0.55; Amano \& Kondo, 2000). Notice that this divergence in frequency should bias Japanese and Dutch infants into opposite directions.

Multiple tokens of /ompa/ and /onta/ were recorded by a female native speaker of Dutch in an infant-directed register. Eight tokens per sequence were selected. These were matched on duration and vowel formant values (cf. Table 1). Five of the eight tokens of each type were used in the labial and coronal habituation lists. Test lists also contained five tokens, of which two had appeared in the habituation lists, and three were novel. This mixture of habituated and novel tokens helps exclude the possibility of dishabituation based on novel tokens alone. Four habituation lists and two test lists were created per sequence by pseudo-randomizing order of tokens. A 1-s pause was inserted between each token, and the mean list length was $14.1 \mathrm{~s}$.

The visual stimulus accompanying auditory stimulus presentation was a dynamic checkerboard presented in 
Table 1

Acoustic characteristics of experimental stimuli.

\begin{tabular}{llll}
\hline & & $\begin{array}{l}\text { /ompa/ mean } \\
\text { (SD) }\end{array}$ & $\begin{array}{l}\text { /onta/ mean } \\
\text { (SD) }\end{array}$ \\
\hline Duration (ms) & & $773(46)$ & $740(16)$ \\
Pitch (Erb) & & $6.56(0.54)$ & $6.18(0.39)$ \\
Formant frequency $V_{1}$ & F1 & $5.66(0.53)$ & $5.65(0.48)$ \\
$\quad$ (Bark) & F2 & $8.45(0.29)$ & $8.75(0.35)$ \\
& F3 & $16.07(0.17)$ & $16.17(0.17)$ \\
Formant frequency $V_{2}$ & F1 & $7.72(0.23)$ & $7.48(0.17)$ \\
$\quad$ (Bark) & F2 & $11.58(0.07)$ & $11.81(0.04)$ \\
& F3 & $5.30(0.18)$ & $5.31(0.29)$ \\
\hline
\end{tabular}

the middle of the screen. Between trials, a video of a flashing red light was displayed as an attention getter. For the pre-and posttest, a colorful smiley was presented accompanied by multiple tokens of the sound $/ \mathrm{ni}: \mathrm{m} /$.

\subsection{Procedure}

A slightly modified version of the Central Fixation paradigm (Werker, Cohen, Lloyd, Casasola, \& Stager, 1998) was used. The infant sat on a caregiver's lap, facing the screen. Sounds were presented from loudspeakers, and the infant's gaze pattern was recorded by a video camera directly below the screen. The caregiver wore sound-attenuating headphones with masking music. The experimenter also wore headphones with masking music (Netherlands) or was in a different room where she could not hear any of the stimuli (Japan). Infants' gaze on or off the screen was coded online by a trained experimenter. The experiment was administered with the software Habit X (Cohen, Atkinson, \& Chaput, 2004), and started with the presentation of the attention getter. Once the infant looked at the screen, the pre-test was initiated, followed by the habituation phase, in which the dynamic checkerboard and habituation stimuli were presented. Stimulus presentation was fixed. The four habituation lists were presented in four different pseudo-random orders across infants. Half of the infants were habituated to lists of /ompa/ tokens, and half of the infants were habituated to lists of /onta/ tokens. The habituation criterion was a decrease to $60 \%$ of the looking time of the first trial, calculated over a sliding window of 4 trials. Once this criterion was reached or the infant reached the maximum trial number of 28 , the test phase started. During the test phase, infants were presented with 4 trials. Half of these were same trials in which a test list with the same type of sequence as during habituation was presented, and half were switch trials in which a test list with the different sequence was presented. The order of presentation was always same-switch-same-switch, followed by the post-test. The experiment was terminated if the infant started crying or fussing extensively. Gaze behavior was recoded offline by a trained coder and entered into further analysis.

\subsection{Results and discussion}

A preliminary t-test examined the difference in habituation times between conditions, finding a tendency for longer habituation times in infants habituated to /onta/ (mean habituation time to /ompa/: $163 \mathrm{~s}$; /onta/: $193 \mathrm{~s}$; $t(62)=-1.78, p=.080)$. This difference did not affect attentiveness across groups: a preliminary ANOVA on looking times in pre-and post-test trials by age and language revealed no significant differences between pre- and post-test trials $[F(1,60)=0.40, p=0.527]$, and no significant interactions with age or language background.

Nevertheless, looking times during habituation were included as a covariate in the following ANCOVA to control for a possible influence of habituation time. The factors were direction (labial to coronal, coronal to labial), agegroup (4 months, 6 months), and language background (Dutch, Japanese). There was a significant main effect of trial type $\left[F(1,56)=4.76, p=.03, \eta^{2}=.024\right]$, with a longer looking time in switch $(m=8.84 \mathrm{~s}, \mathrm{SD}=2.98)$ than in same trials $(m=8.02 \mathrm{~s}, \mathrm{SD}=2.64)$ and a significant interaction between trial type and direction $[F(1,56)=10.97, p=.001$, $\left.\eta^{2}=.054\right]$. No other effects approached significance; in particular, although there was a marginal effect of language background $\left[F(1,56)=3.64, p=.061, \eta^{2}=.043\right]$, the interaction term between language background and trial type was not significant $\left[F(1,56)=0.70, p=.406, \eta^{2}=.003\right]$ nor was the three-way interaction among language background, trial type, and direction $[F(1,56)=0.02, p=.891$, $\left.\eta^{2}=.0001\right]$.

Bonferroni-corrected post hoc t-tests following up on the interaction between trial type and direction indicated that the effect of trial type was only significant for the direction labial to coronal $\left(m_{\text {same }}=7.61 \mathrm{~s}, m_{\text {switch }}=9.68 \mathrm{~s}\right.$, $p=.019, d=1.23)$, but not vice versa $\left(m_{\text {same }}=8.42 \mathrm{~s}\right.$, $\left.m_{\text {switch }}=7.99 \mathrm{~s}, p=1.00, d=-0.26\right)$. For the labial to coronal direction 24 out of 32 infants (75\%) looked longer to 'switch' than to 'same' trials, while that was only true for 15 out of 32 infants (47\%) for the direction coronal to labial.

Breaking down effect sizes by language and direction indicated comparable effect sizes across languages (labial to coronal, Dutch: $d=1.39$, Japanese: $d=1.14$; coronal to labial, Dutch: $d=-0.09$, Japanese: $d=-0.45$ ) (see Fig. 1 ).

\section{General discussion}

The current study found evidence for a perceptual directional asymmetry in a word-medial labial-coronal consonant contrast for 4- and 6-month-old Dutch and Japanese infants: they were able to perceive the difference between labial and coronal consonants if habituated to labial, but not if habituated to coronal consonants. In other words, labial tokens were accepted as instances of coronals, but not vice versa. The results support a languageindependent account of the coronal-labial perceptual asymmetry, present prior to the age where infants' perception is tuned to language-specific phonological characteristics. Previous work has found effects of frequency, but only towards the end of the first year of life (at 9-12 months; e.g. Anderson, Morgan, \& White, 2003; Pons, AlbaredaCastellot, \& Sebastián-Gallés, 2012). In addition, the phonotactic frequency of the experimental tokens would have biased infants in opposite directions, suggesting the asymmetry was independent of infants' language background. 

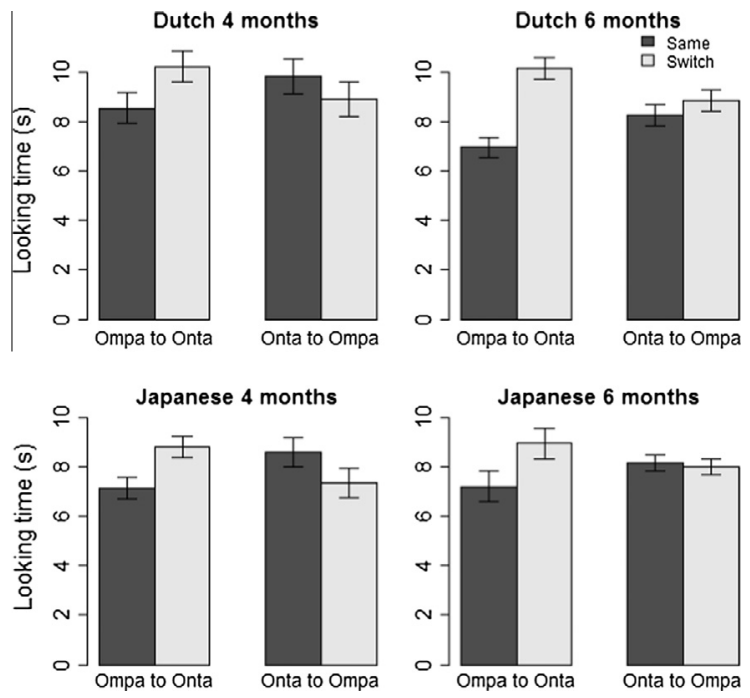

Fig. 1. Looking times to same and switch trials by direction of presentation, language background, and age.

Although neither FUL nor the Optimal Perception account are intended to account for prelexical infants' behavior, the present results are clearly inconsistent with the Optimal Perception account. It should, however, be noted that our results do not preclude the possibility that perception could be modulated by the phonotactic probabilities of the ambient language later on. Instead, the presence of an asymmetry in infants in both languages is not inconsistent with the predictions of FUL either (Lahiri \& Reetz, 2010), although it is unclear in what way the lexical level representations assumed in FUL would translate to the present results with prelexical infants.

Given that the results of the present study strongly suggest that the labial-coronal asymmetry is present independent of language-specific experience, the next question concerns the origins of this perceptual bias. Although it is clearly beyond the scope of the current article to address this question conclusively, we offer speculations on two possible perceptually-driven mechanisms; namely differences in the individual and/or distributional acoustic properties of coronal and labial tokens. The former is rooted in previous psychological work revealing that feature omissions often lead to misperception whereas misperception due to feature additions are rare (e.g. Q is misperceived as $\mathrm{O}$ but the reverse is rare; Gilmore, Hersh, Caramazza, \& Griffin, 1979). Based on such work, we can reason that a similar process could explain asymmetries between / $t$ / and $/ \mathrm{p} /$ : the burst of $/ \mathrm{t} / \mathrm{has}$ a high frequency energy compared to $/ \mathrm{p} /$, which could lead to perceptual asymmetries. When listening to $/ \mathrm{t} /$ in succession to $/ \mathrm{p} /$, listeners get clear evidence for a change based on the added noisy / $t$ / burst, but when listening to $/ \mathrm{p} /$ after $/ \mathrm{t} /$, they might think that they missed the noisy burst in the second sound. Asymmetries in consonant perception based on such differences in acoustic characteristics have been demonstrated by Chang, Plauché, and Ohala (2001).

Additionally (or alternatively), the asymmetry could be caused not by the intrinsic properties of each token, but by the acoustic distributions of the group of tokens. In the domain of visual categorization, 3-to 4-month-old infants have been shown to discriminate cats and dogs in an asymmetric fashion based on the category variability they were exposed to during an experiment: when the category variability of dog faces was higher than of cat faces, infants failed to detect a cat face. This effect disappeared when variability was matched across categories (Quinn, Eimas, \& Rosenkrantz, 1993). Similarly, coronal tokens, which depend on a closure that often varies in exact position and extent within and across talkers, might show a higher variability than labial ones. Such a mechanism could either operate based on variability experienced during the experiment, or on previous experience with variability in the ambient language.

We examined the standard deviations of acoustic measurements relevant to place of articulation in our stimuli as one possible index of the first type of variability (such as F2 values at vowel-consonant juncture, Narayan, 2008, or skewness of plosive burst, Stevens \& Blumstein, 1978, cf. Supplementary Material). These do not, however, support such an account, since measures related to F2 at juncture showed larger standard deviations in coronal compared to labial tokens, while the reverse is true for measures related to the burst. To investigate the second type of variability, a more in-depth study of the acoustic properties of labial and coronal consonants in both elicited and natural spoken language corpora is necessary.

Asymmetries in prelexical infants' speech perception have played a major role in vowel discrimination research (see Polka \& Bohn, 2011). However, the mechanisms proposed to underlie this perceptual bias (Schwartz, Abry, Boe, Menard, \& Vallee, 2005) cannot easily be extrapolated to consonants, which are characterized by very different acoustic properties.

In conclusion, our data provide evidence for an early and language-general labial-coronal perceptual asymmetry, contributing a critical piece of evidence to our understanding of how the special status of coronals is reflected in human speech perception. The observed early asymmetries are unlikely to be anchored in lexical representations or caused by phonotactic probabilities. Therefore, our data place new constraints on theories of speech perception by requiring them to take into account perceptual biases at the level of sounds (prior to other biases that may emerge from the phonotactics or the lexicon of a language with exposure). In addition, our findings direct further research into the origins of the labial-coronal perceptual asymmetry by suggesting that these biases likely emerge from the acoustic characteristics of sounds. Further research will not only shed light on the origins of the special role of coronals in the languages of the world, but also speak to the goal of a perceptually grounded theory of infant speech perception.

\section{Acknowledgments}

We want to thank Yuri Hatano, Naoko Kijima, Dirkje van der Aa and Karin van Usen for their assistance with recruiting and testing infants, Imme Lammertink and Risa Oomori for the cross-coding of infant videos, and Aditi 
Lahiri for helpful discussion. This work was supported by an IMPRS for Language Sciences fellowship and a RIKEN short-term IPA fellowship to the first author. AC acknowledges the institutional support from ANR-10-LABX-0087 IEC and ANR-10-IDEX-0001-02 PSL*.

\section{Appendix A. Supplementary material}

Supplementary data associated with this article can be found, in the online version, at http://dx.doi.org/10.1016/ j.cognition.2014.10.009.

\section{References}

Amano, S., \& Kondo, T. (2000). Nihongo-no Goi-tokusei [Lexical properties of Japanese] (Vol. 7). Tokyo: Sansei-do.

Anderson, J. L., Morgan, J. L., \& White, K. S. (2003). A statistical basis for speech sound discrimination. Language and Speech, 46(2-3), 155-182. http://dx.doi.org/10.1177/00238309030460020601.

Baayen, R. H., Piepenbrock, R., \& Gulikers, L. (1995). The CELEX lexical database (CD-ROM). Philadelphia, PA: Linguistic Data Consortium, University of Pennsylvania.

Bergelson, E., \& Swingley, D. (2012). At 6-9 months, human infants know the meaning of many common nouns. PNAS, 109(9), 3253-3258. http://dx.doi.org/10.1073/pnas.1113380109.

Bonte, M., Mitterer, H., Zellagui, N., Poelmans, N., \& Blomert, L. (2005) Auditory cortical tuning to statistical regularities in phonology. Clinical Neurophysiology, 116, 2765. http://dx.doi.org/10.1016/ j.clinph.2005.08.012.

Chang, S., Plauché, M. C., \& Ohala, J. J. (2001). Markedness and consonant confusion asymmetries. In E. Hume \& K. Johnson (Eds.), The role of speech perception in phonology (pp. 79-101). San Diego, CA: Academic Press.

Chomsky, N., \& Halle, M. (1968). The sound pattern of English. New York: Harper and Row.

Cohen, L. B., Atkinson, D. J., \& Chaput, H. H. (2004). Habit X: A new program for obtaining and organizing data in infant perception and cognition studies (Version 1.0). Austin: University of Texas.

Cornell, S. A., Lahiri, A., \& Eulitz, C. (2013). Inequality across consonantal contrasts in speech perception: Evidence from mismatch negativity. Journal of Experimental Psychology: Human Perception and Performance, 39(3), 757-772. http://dx.doi.org/10.1037/a0030862.

Dijkstra, N., \& Fikkert, P. (2011). Universal constraints on the discrimination of Place of Articulation? Asymmetries in the discrimination of 'paan' and 'taan' by 6-month-old Dutch infants. In N. Danis, K. Mesh, \& H. Sung (Eds.). Proceedings of the Boston University conference on language development (Vol. 35, pp. 170-182).
Gilmore, G. C., Hersh, H., Caramazza, A., \& Griffin, J. (1979). On the prediction of confusion matrices from similarity judgments. Perception E' Psychophysics, 25(5), 425-431.

Jusczyk, P. W., Smolensky, P., \& Alloco, T. (2002). How English-learning infants respond to markedness and faithfulness constraints. Language Acquisition, 10(1), 31-73.

Kuhl, P. K. (2004). Early language acquisition: Cracking the speech code. Nature Reviews Neuroscience, 5, 831-843. http://dx.doi.org/10.1038/ nrn1533.

Lahiri, A., \& Reetz, H. (2010). Distinctive features: Phonological underspecification in processing. Journal of Phonetics, 38, 44-59. http://dx.doi.org/10.1016/j.wocn.2010.01.002.

Lahiri, A., \& Reetz, H. (2002). Underspecified recognition. In C. Gussenhoven \& N. Warner (Eds.), Laboratory phonology VII (pp. 637-677). Berlin, Germany: Mouton de Gruyter.

Maddiesson, I. (1984). Patterns of sounds. New York: Cambridge University Press.

Mitterer, H. (2011). The mental lexicon is fully specified: Evidence from eye-tracking. Journal of Experimental Psychology: Human Perception and Performance, 37, 496-513. http://dx.doi.org/10.1037/a0020989.

Narayan, C. R. (2008). The acoustic-perceptual salience of nasal place contrasts. Journal of Phonetics, 36, 191-217. http://dx.doi.org/10.1016/ j.wocn.2007.10.001.

Paradis, C., \& Prunet, J.-F. (Eds.). (1991). The special status of coronals: Internal and external evidence (Phonetics and Phonology 2). San Diego: Academic Press.

Polka, L., \& Bohn, O.-S. (2011). Natural Referent Vowel (NRV) framework: An emerging view of early phonetic development. Journal of Phonetics, 39, 467-478. http://dx.doi.org/10.1016/j.wocn.2010.08.007.

Pons, F., Albareda-Castellot \& Sebastián-Gallés, N. (2012). The interplay between input and initial biases: Asymmetries in vowel perception during the first year of life. Child Development, 83(3), 965-976. http:// dx.doi.org/10.1111/j.1467-8624.2012.01740.x.

Quinn, P. C., Eimas, P. D., \& Rosenkrantz, S. L. (1993). Evidence for representations of perceptually similar natural categories by 3month-old and 4-month-old infants. Perception, 22(4), 463-475. http://dx.doi.org/10.1068/p220463.

Schwartz, J.-L., Abry, C., Boe, L.-J., Menard, L., \& Vallee, N. (2005) Asymmetries in vowel perception, in the context of the DispersionFocalisation Theory. Speech Communication, 45, 425-434. http:// dx.doi.org/10.1016/j.specom.2004.12.001.

Stevens, K. N., \& Blumstein, S. E. (1978). Invariant cues for place of articulation in stop consonants. The Journal of the Acoustical Society of America, 64(5), 1358-1368.

Tsuji, S., Nishikawa, K., \& Mazuka, R. (2010). Input frequency of place of articulation in Japanese infant-directed speech: An exceptional case. In Poster presented at the 17th international conference on infant studies, Baltimore, USA.

Werker, J. F., Cohen, L. B., Lloyd, V. L., Casasola, M., \& Stager, C. L. (1998). Acquisition of word-object associations by 14-month-old infants. Developmental Psychology, 34(6), 1289-1309. http://dx.doi.org 10.1037/0012-1649.34.6.1289. 